\title{
Spatial Peak Power Minimization for Relaxed Phase $M$-PSK MIMO Directional Modulation Transmitter
}

\author{
Ashkan Kalantari, Christos Tsinos, Mojtaba Soltanalian, \\ Symeon Chatzinotas, Wing-Kin Ma, and Björn Ottersten
}

\begin{abstract}
The burst in media content and access to smart phones has created an increasing demand for data. At the same time, powering up mobile base stations contributes notably to $\mathrm{CO}_{2}$ footprint. To address these issues, we need to design energy efficient communication systems with higher data rates while considering practical limitations. As a solution, we design an optimal $M$-PSK directional modulation precoder with spatial peak power minimization where the communicated symbol on each receiving antenna is placed in the optimal location of a predefined region. Such an approach allows less stringent design and results in further energy efficiency. In this work, we characterize the relaxed region, formulate the optimal symbollevel precoder design problem, and transform it into a standard form. The simulation results show that the relaxed design reduces the consumed power while the symbol error rate increment at the receiver due to the relaxed phase design is negligible.
\end{abstract}

Keywords-Directional modulation, energy efficiency, M-PSK modulation, spatial peak power, symbol-level precoding

\section{INTRODUCTION}

The advent of mobile smart devices, e.g., smart phones and tablets, and the boom of Internet content, has led to a fast growth in users' demand for data communication. Based on Cisco's white paper [1], it is predicted that the mobile Internet traffic will increase eight folds from 2015 to 2020. Therefore, it is necessary to effectively use the available resources in order to address the increasing rate demand. Other than time and frequency resources [2], [3], precoding at the transmitter [4], [5] in order to use the spatial dimension has shown a significant potential to improve the data rate by reducing the interference and satisfying the quality of service at the users. As another paradigm to combat the interference, directional modulation [6]-[9] and constructive interference [10]-[12] have been developed to communicate multiple interference-limited or interference-free streams with the receiver. In directional modulation, the antenna weights are designed such that the phase and signal-to-noise ratio (SNR) of the receiving signal on each antenna of the receiver are respectively equivalent to the phase and SNR of a specific desired symbol. In fact, in the directional modulation, the modulation happens while the radio frequency (RF) signal passes through the fading channel.

\footnotetext{
This work was supported by the National Research Fund (FNR) of Luxembourg under AFR grant for the project SeMIGod. Ashkan Kalantari, Christos Tsinos, Symeon Chatzinotas, and Björn Ottersten are with the Interdisciplinary Centre for Security, Reliability and Trust (SnT), The University of Luxembourg, 29, avenue JF Kennedy, L-1855 Luxembourg-Kirchberg, Luxembourg, (E-mails: \{ashkan.kalantari,christos.tsinos,symeon.chatzinotas,bjorn.ottersten\}@uni.lu) Mojtaba Soltanalian is with the Department of Electrical and Computer Engineering, University of Illinois at Chicago, Chicago, IL 60607, E-mail: (msol@uic.edu). Wing-Kin $\mathrm{Ma}$ is with the Department of Electronic Engineering, The Chinese University of Hong Kong, Shatin, Hong Kong, China (e-mail:wkma@ieee.org).
}

In the architecture of the directional, the power of the $\mathrm{RF}$ oscillator is equally divided among the RF chains [6][9] and the power amplifier in each RF chain needs to operate in a specific range to avoid nonlinear distortion of the amplified signal [13]. To keep the amplifier in the linear region, we need to consider an upper limit on the output power of the amplifier in each RF chain. As a solution, we can design a directional modulation transmitter with spatial peak power minimization. To this end, the references [14], [15] consider constant envelope precoding for a single-user massive MIMO system and the authors in [16] consider a perantenna power minimization based on constructive interference where there is a strict constraint on the phase of the received symbols. In addition to the hardware considerations, the mobile communications consume a large amount of energy which is responsible for a considerable amount of environmental pollution [17]. Reducing the energy consumed at the radio access networks is not only environmental friendly, but also it reduces the mobile communications cost. The works of [9][11], [18], [19] consider a relaxed design approach to improve the energy efficiency at the transmitter.

Based on the above descriptions, it is in the best interest of both mobile operators and users to have a system which jointly takes into account the increasing rate demand, hardware limitation, and energy efficiency. A constant envelop design such as [14], [15] results in non-convex problems and conservative designs. Also, the constructive interference approach in [16] considers a fixed phase design for low peak power to average ratio, which results in a stringent design. However, there is no work on addressing a joint design considering both amplifier distortion and energy efficiency. To address this, we design an $M$-PSK directional modulation transmitter with spatial peak power minimization while a relaxed region for further energy efficiency of $M$-PSK symbol is considered. In this relaxed design, the $M$-PSK symbol can be placed in the optimal location of a defined region. This results in lower power consumption while satisfying the SNR requirement at the receiver.

The remainder of this paper is organized as follows. In Section II, the signal model is introduced. The spatial peak power minimization problems for the fixed and relaxed designs are formulated into standard forms in Section III. In Section IV, we present simulation results by comparing the proposed method with the benchmark schemes and draw conclusions in Section V.

Notation: Upper-case and lower-case bold-faced letters are used to denote matrices and column vectors, respectively. The superscripts $(\cdot)^{T},(\cdot)^{*},(\cdot)^{H}$, and $(\cdot)^{\dagger}$ represent transpose, 
conjugate, Hermitian, and Moore-Penrose pseudo inverse operators, respectively. $\mathbf{I}_{N \times N}$ denotes an $N$ by $N$ identity matrix, $\mathbf{E}_{k}$ has one unit-valued element on the $k$-th diagonal entry with the rest of the elements being zero, $\widetilde{\mathbf{E}}_{k}$ has two unitvalued elements on the $k$-th and $\left(N_{t}+k\right)$-th diagonal entries with the rest of the elements being zero, $\operatorname{diag}(\mathbf{a})$ denotes a diagonal matrix where the elements of the vector a are its diagonal entries, $\mathbf{a} \circ \mathbf{b}$ is the element-wise Hadamard product, $\mathbf{0}$ is the all zero vector, $\|\cdot\|$ is the Frobenius norm, and $|\cdot|$ represents the absolute value of a scalar. $\operatorname{Re}(\cdot), \operatorname{Im}(\cdot)$, and $\arg (\cdot)$ represent the real part, imaginary part, and angle of a complex number, respectively.

\section{Signal And System Model}

Consider a single carrier transmitter with $N_{t}$ antennas, denoted by $T$, that functions using the directional modulation concept and communicates with a receiver with $N_{r}$ antennas, denoted by $R$. The received signal, $\mathbf{y}$, at the receiver can be written as

$$
\mathbf{y}=\mathbf{H w}+\mathbf{n},
$$

where $\mathbf{y}$ is an $N_{r} \times 1$ vector denoting the received signals by $R, \mathbf{H}=\left[\mathbf{h}_{1}, \ldots, \mathbf{h}_{n}, \ldots, \mathbf{h}_{N_{r}}\right]^{T}$ is an $N_{r} \times N_{t}$ matrix denoting the channel from $T$ to $R, \mathbf{h}_{n}$ is an $N_{t} \times 1$ vector containing the channel coefficients from the transmitter antennas to the $n$-th antenna of $R$, and $\mathbf{w}=\left[w_{1}, \ldots, w_{n}, \ldots, w_{N_{t}}\right]$ is the transmit vector. The random variable $\mathbf{n} \sim \mathcal{C N}\left(\mathbf{0}, \sigma^{2} \mathbf{I}_{N_{r} \times N_{\mathcal{N}}}\right)$ denotes the additive white Gaussian noise at $R$ where $\mathcal{C} \mathcal{N}$ denotes a complex and circularly symmetric random variable. In the directional modulation, the elements of $\mathbf{H w}=$ $\left[s_{1}^{\prime}, \ldots, s_{n}^{\prime}, \ldots, s_{N_{r}}^{\prime}\right]^{T}$ are the induced $M$-PSK symbols on the antennas of $R$ where $s_{n}^{\prime}$ is the induced $M$-PSK symbol on the $n$-th antenna of $R, \mathbf{s}=\left[s_{1}, \ldots, s_{n}, \ldots, s_{N_{r}}\right]$ are the $M$ PSK symbols to be communicated between $T$ and $R$ with instantaneous unit energy, i.e., $\left|s_{n}\right|^{2}=1$. To detect the received symbols, $R$ can apply conventional detectors on each receiving antenna.

If we show the instantaneous output signal in the $n$-th RF chain as $w_{n}$, the maximum output power among the RF chains can be defined as

$$
P_{\max }^{\text {spatial }}=\max _{k=1, \ldots, N_{t}} \mathbf{w}^{H} \mathbf{E}_{k} \mathbf{w},
$$

which we refer to it as the spatial peak power. In the next section, we aim to minimize the spatial peak power.

\section{Spatial Peak Power Minimization for DiRECTIONAL MODULATION}

In this section, we formulate the fixed benchmark and relaxed designs with the goal of spatial peak power minimization for a $M$-PSK directional modulation transmitter and design the optimal symbol-level precoder for each case.

\section{A. Peak Power Minimization: Fixed Design}

In this section, we formulate and transform the $M$-PSK precoder design problem that satisfies the exact required phase and the minimum required SNR for the signal received on each antenna of $R$, the induced desired symbol, while minimizing the spatial peak power. Since a fixed phase is required at the destination, increasing the required signal level at the receiver results in a proportional increment in real and imaginary parts of the received signal. Hence, we can break the SNR constraint into two constraints on real and imaginary parts of the induced $M$-PSK symbol, $s_{n}$, as

$$
\operatorname{Re}\left(\mathbf{h}_{n}^{T} \mathbf{w}\right) \geq \sqrt{\gamma} \operatorname{Re}\left(s_{n}\right), \operatorname{Im}\left(\mathbf{h}_{n}^{T} \mathbf{w}\right) \geq \sqrt{\gamma} \operatorname{Im}\left(s_{n}\right),
$$

where $\gamma$ is the required signal power at the receiver defined as $\gamma=10^{S N R / 10}$. Putting down the SNR constraint as in (3) helps us avoid a non-convex design. Since the phase of the received signal is fixed, we only need to use either the real or imaginary part in (3). Using (3), the design problem is cast as

$$
\begin{aligned}
\min _{\mathbf{w}, t} & \mathbf{w}^{H} \mathbf{E}_{k} \mathbf{w} \\
\text { s.t. } & \arg \left(\mathbf{h}_{n}^{T} \mathbf{w}\right)=\arg \left(s_{n}\right), \forall n=1, \ldots, N_{r} \\
& \operatorname{Re}\left(s_{n}\right) \operatorname{Re}\left(\mathbf{h}_{n}^{T} \mathbf{w}\right) \geq \sqrt{\gamma} \operatorname{Re}^{2}\left(s_{n}\right) . \forall n=1, \ldots, N_{r}
\end{aligned}
$$

The constraint (3), will not satisfy the signal level requirement if $\operatorname{Re}\left(\mathbf{h}_{n}^{T} \mathbf{w}\right)<0$. To address this issue, both sides of $(4 \mathrm{~b})$ are multiplied by $\operatorname{Re}\left(s_{n}\right)$. This does not affect the inequality since $\operatorname{Re}\left(\mathbf{h}_{n}^{T} \mathbf{w}\right)$ and $\operatorname{Re}\left(s_{n}\right)$ have the same sign at the optimal point. By introducing $t$ as an auxiliary variable, we can transform (4) into a more familiar form as

$$
\begin{array}{ll}
\min _{\mathbf{w}, t} & t \\
\text { s.t. } & \mathbf{w}^{H} \mathbf{E}_{k} \mathbf{w} \leq t, \forall k=1, \ldots, N_{t} \\
& \arg \left(\mathbf{h}_{n}^{T} \mathbf{w}\right)=\arg \left(s_{n}\right), \forall n=1, \ldots, N_{r} \\
& \operatorname{Re}\left(s_{n}\right) \operatorname{Re}\left(\mathbf{h}_{n}^{T} \mathbf{w}\right) \geq \sqrt{\gamma} \operatorname{Re}^{2}\left(s_{n}\right) . \forall n=1, \ldots, N_{r}
\end{array}
$$

We can write the phase constraint in (5b) in the linear from as $\alpha_{n} \operatorname{Re}\left(\mathbf{h}_{n}^{T} \mathbf{w}\right)-\operatorname{Im}\left(\mathbf{h}_{n}^{T} \mathbf{w}\right)=0$ and stack the constraints sets in (5b) and (5c) to get

$$
\begin{array}{ll}
\min _{\mathbf{w}, t} & t \\
\text { s.t. } & \mathbf{w}^{H} \mathbf{E}_{k} \mathbf{w} \leq t, \\
& \mathbf{A R e}(\mathbf{H w})-\operatorname{Im}(\mathbf{H w})=\mathbf{0}, \\
& \operatorname{Re}(\mathbf{S}) \operatorname{Re}(\mathbf{H w}) \geq \sqrt{\gamma} \mathbf{s}_{r},
\end{array}
$$

where $\mathbf{S}=\operatorname{diag}(\mathbf{s})$, $\mathbf{s}$ is an $N_{r} \times 1$ vector containing the $M$-PSK symbols to be communicated, $\mathbf{s}_{r}=\operatorname{Re}(\mathbf{s}) \circ \operatorname{Re}(\mathbf{s})$, $\mathbf{A}=\operatorname{diag}(\boldsymbol{\alpha}), \boldsymbol{\alpha}=\left[\alpha_{1}, \ldots, \alpha_{n}, \ldots, \alpha_{N_{r}}\right]^{T}$, and $\alpha_{n}=$ $\tan \left(\arg \left(s_{n}\right)\right)=\operatorname{Im}\left(s_{n}\right) / \operatorname{Re}\left(s_{n}\right)$. To remove the real and imaginary parts from (6), we can use the results of [9] as

$$
\operatorname{Re}(\widetilde{\mathbf{H}} \mathbf{w})=\mathbf{H}_{1} \widetilde{\mathbf{w}}, \operatorname{Im}(\widetilde{\mathbf{H}} \mathbf{w})=\mathbf{H}_{2} \widetilde{\mathbf{w}}
$$

where $\widetilde{\mathbf{w}}=\left[\operatorname{Re}\left(\mathbf{w}^{T}\right), \operatorname{Im}\left(\mathbf{w}^{T}\right)\right]^{T}, \quad \mathbf{H}_{1}=$ $[\operatorname{Re}(\widetilde{\mathbf{H}}),-\operatorname{Im}(\widetilde{\mathbf{H}})], \quad \mathbf{H}_{2}=[\operatorname{Im}(\widetilde{\mathbf{H}}), \operatorname{Re}(\widetilde{\mathbf{H}})]$, and $\|\widetilde{\mathbf{w}}\|^{2}=\|\mathbf{w}\|^{2}$. Using (7), (6) transforms into

$$
\begin{array}{ll}
\min _{\widetilde{\mathbf{w}}, t} & t \\
\text { s.t. } & \widetilde{\mathbf{w}}^{T} \widetilde{\mathbf{E}}_{k} \widetilde{\mathbf{w}} \leq t, \\
& \left(\mathbf{A} \mathbf{H}_{1}-\mathbf{H}_{2}\right) \widetilde{\mathbf{w}}=\mathbf{0}, \\
& \operatorname{Re}(\mathbf{S}) \mathbf{H}_{1} \widetilde{\mathbf{w}} \geq \sqrt{\gamma} \mathbf{s}_{r} .
\end{array}
$$

Similar as in [9], we can put $\mathbf{w}$ in the null space of $\mathbf{A} \mathbf{H}_{U_{1}}-\mathbf{H}_{U_{2}}$. If the SVD of $\mathbf{A} \mathbf{H}_{1}-\mathbf{H}_{2}$ is given by $\mathbf{U} \Sigma \mathbf{V}^{H}$, 


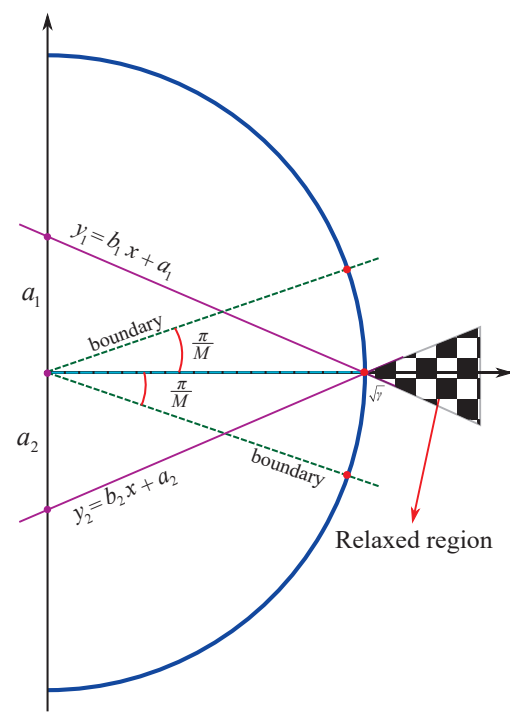

Fig. 1. Characterization of the relaxed region for $M$-PSK modulation.

the orthonormal basis for the null space of $\mathbf{A} \mathbf{H}_{1}-\mathbf{H}_{2}$ are the last $2 N_{t}-r^{\prime}$ columns of the matrix $\mathbf{V}$ with $r^{\prime}$ being the rank of $\mathbf{A H}_{1}-\mathbf{H}_{2}$ [20]. Using this, we can write $\widetilde{\mathbf{w}}$ as $\widetilde{\mathbf{w}}=\mathbf{V} \boldsymbol{\lambda}$ where

$$
\mathbf{V}=\left[\mathbf{v}_{r^{\prime}+1}, \ldots, \mathbf{v}_{2 N_{t}}\right], \boldsymbol{\lambda}=\left[\lambda_{1}, \ldots, \lambda_{2 N_{t}-r^{\prime}}\right] .
$$

By replacing $\widetilde{\mathbf{w}}$ with $\mathbf{V} \boldsymbol{\lambda}$, (8) reduces into

$$
\begin{array}{ll}
\min _{\boldsymbol{\lambda}, t} & t \\
\text { s.t. } & \widetilde{\boldsymbol{\lambda}}^{T} \mathbf{B} \widetilde{\boldsymbol{\lambda}} \leq t, \\
& \mathbf{C} \boldsymbol{\lambda} \geq \mathbf{a},
\end{array}
$$

where $\mathbf{B}=\mathbf{V}^{T} \widetilde{\mathbf{E}}_{i} \mathbf{V}, \mathbf{C}=\operatorname{Re}(\mathbf{S}) \mathbf{H}_{1} \mathbf{V}$, and $\mathbf{a}=\sqrt{\gamma} \mathbf{s}_{r}$. The design in (10) is a linear program with quadratic and linear constraints which can be solved via standard approaches.

\section{B. Energy Efficient Peak Power Minimization: Relaxed Design}

In this part, we design the optimal spatial peak power minimization precoder for directional modulation transmitter by letting the phase of the received signal on each antenna of $R$ vary in a defined region, shown by check board pattern in Fig. 1. This results in a less stringent design compared to [14][16] and consequently reduces the power consumption at the transmitter.

To do so, first, we characterize the relaxed phase region in which the phase of the receiving signal on each antenna of $R$ can vary. For an easy design, we characterize the relaxed region on the real axis, as shown in Fig. 1, and then rotate the other symbols into this region and apply the defined constraints on them. In $M$-PSK, each symbol has a detection region within $\frac{\pi}{M}$ degrees from each direction. Also, considering that we want to satisfy a specific signal level, $\sqrt{\gamma}$, for each induced symbol at the receiver, we need to consider the relaxed region outside the circle of Fig. 1. One way to satisfy the required SNR and leaving enough distance to the boundaries of the detection region is drawing lines $y_{1}$ and $y_{2}$ parallel with the detection region boundary so that these lines pass through the signal level threshold, $\sqrt{\gamma}$, as illustrated in Fig. 1.
After defining the boundaries of the relaxed region, we extend it to other symbols. As the first step to define the relaxed region for the symbol $s_{n}$, we need to multiply it by $e^{-i \varphi_{n}}$ so that it rotates into the defined relaxed region of Fig. 1 shown by the check board pattern. The terms $\operatorname{Re}\left(\mathbf{h}_{n}^{T} \mathbf{w}\right)$ and $\operatorname{Im}\left(\mathbf{h}_{n}^{T} \mathbf{w}\right)$ are equivalent to $x$ and $y$ in the 2D plane of Fig. 1, therefore, the relaxed region of symbol $s_{n}$ can be defined using the following two inequalities:

$$
\begin{gathered}
b_{1} \operatorname{Re}\left(\mathbf{h}_{n}^{T} \mathbf{w} e^{-i \varphi_{n}}\right)+a_{1} \leq \operatorname{Im}\left(\mathbf{h}_{n}^{T} \mathbf{w} e^{-i \varphi_{n}}\right), \\
\operatorname{Im}\left(\mathbf{h}_{n}^{T} \mathbf{w} e^{-i \varphi_{n}}\right) \leq b_{2} \operatorname{Re}\left(\mathbf{h}_{n}^{T} \mathbf{w} e^{-i \varphi_{n}}\right)+a_{2} .
\end{gathered}
$$

The parameters of lines $y_{1}$ and $y_{2}$ can be defined as

$$
\begin{array}{ll}
b_{1}=-\tan \left(\frac{\pi}{M}\right), b_{2}=\tan \left(\frac{\pi}{M}\right), \\
a_{1}=-b_{1} \sqrt{\gamma}, \quad a_{2}=-b_{2} \sqrt{\gamma} .
\end{array}
$$

Using the defined relaxed region in (11), the relaxed optimal precoder design problem is defined as

$$
\begin{array}{ll}
\min _{\mathbf{w}, t} & t \\
\text { s.t. } & \mathbf{w}^{H} \mathbf{E}_{k} \mathbf{w} \leq t, \quad \forall k=1, \ldots, N_{t} \\
& \operatorname{Im}\left(\mathbf{h}_{n}^{T} \mathbf{w} e^{-i \varphi_{n}}\right) \geq b_{1} \operatorname{Re}\left(\mathbf{h}_{n}^{T} \mathbf{w} e^{-i \varphi_{n}}\right)+a_{1}, \\
& \operatorname{Im}\left(\mathbf{h}_{n}^{T} \mathbf{w} e^{-i \varphi_{n}}\right) \leq b_{2} \operatorname{Re}\left(\mathbf{h}_{n}^{T} \mathbf{w} e^{-i \varphi_{n}}\right)+a_{2},
\end{array}
$$

where a similar approach the same as getting from (5) to (6) is used to get to (13). The constraint (13a) limits the instantaneous power transmitted from each RF chain. The constraints (13b) and (13c) enforce the induced symbol to be within the relaxed region while satisfying the minimum required SNR at the receiver. As the first step to simplify (13), we absorb the desired symbol phase $e^{-i \varphi_{n}}$ into the channel to get

$$
\begin{array}{ll}
\min _{\mathbf{w}, t} & t \\
\text { s.t. } & \mathbf{w}^{H} \mathbf{E}_{k} \mathbf{w} \leq t, \\
& \operatorname{Im}\left(\widetilde{\mathbf{h}}_{n}^{T} \mathbf{w}\right) \geq b_{1} \operatorname{Re}\left(\widetilde{\mathbf{h}}_{n}^{T} \mathbf{w}\right)+a_{1}, \\
& \operatorname{Im}\left(\widetilde{\mathbf{h}}_{n}^{T} \mathbf{w}\right) \leq b_{2} \operatorname{Re}\left(\widetilde{\mathbf{h}}_{n}^{T} \mathbf{w}\right)+a_{2},
\end{array}
$$

where $\widetilde{\mathbf{h}}_{n}^{T}=\mathbf{h}_{n}^{T} e^{-i \varphi_{n}}$. Then, we can stack the constraint in $(14 b)$ and $(14 c)$ to get

$$
\begin{array}{ll}
\min _{\mathbf{w}, t} & t \\
\text { s.t. } & \mathbf{w}^{H} \mathbf{E}_{k} \mathbf{w} \leq t, \\
& \operatorname{Im}(\widetilde{\mathbf{H}} \mathbf{w}) \geq b_{1} \operatorname{Re}(\widetilde{\mathbf{H}} \mathbf{w})+a_{1} \mathbf{1}, \\
& \operatorname{Im}(\widetilde{\mathbf{H}} \mathbf{w}) \leq b_{2} \operatorname{Re}(\widetilde{\mathbf{H}} \mathbf{w})+a_{2} \mathbf{1},
\end{array}
$$

where $\widetilde{\mathbf{H}}=\left[\widetilde{\mathbf{h}}_{1}, \ldots, \widetilde{\mathbf{h}}_{n}, \ldots, \widetilde{\mathbf{h}}_{N_{r}}\right]^{T}$ and $\mathbf{1}$ is an $N_{r} \times 1$ vector containing unit-valued elements. Using derivation in (7), we can remove the real and imaginary parts of (15) to get

$$
\begin{array}{ll}
\min _{\widetilde{\mathbf{w}}, t} & t \\
\text { s.t. } & \widetilde{\mathbf{w}}^{T} \widetilde{\mathbf{E}}_{k} \widetilde{\mathbf{w}} \leq t, \\
& \mathbf{D} \widetilde{\mathbf{w}} \geq \mathbf{d},
\end{array}
$$




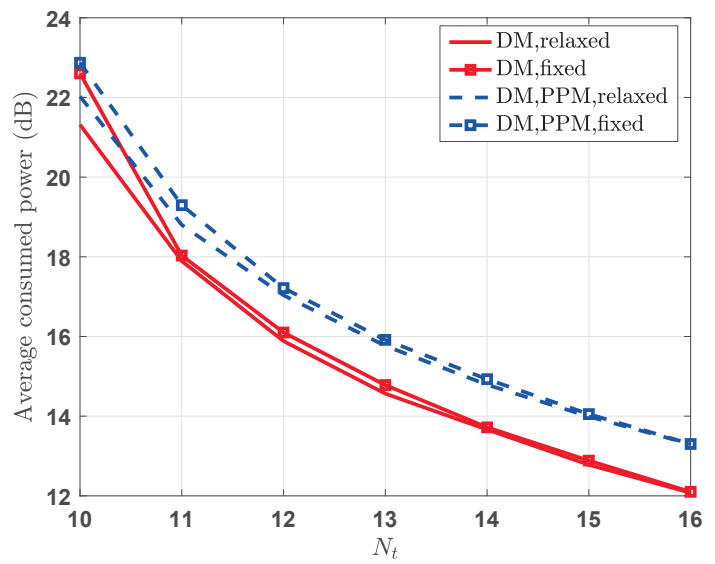

Fig. 2. Average consumed power with respect to $N_{t}$ for the proposed and benchmark schemes with $S N R=10 \mathrm{~dB}, N_{r}=10$, and $M=16$.

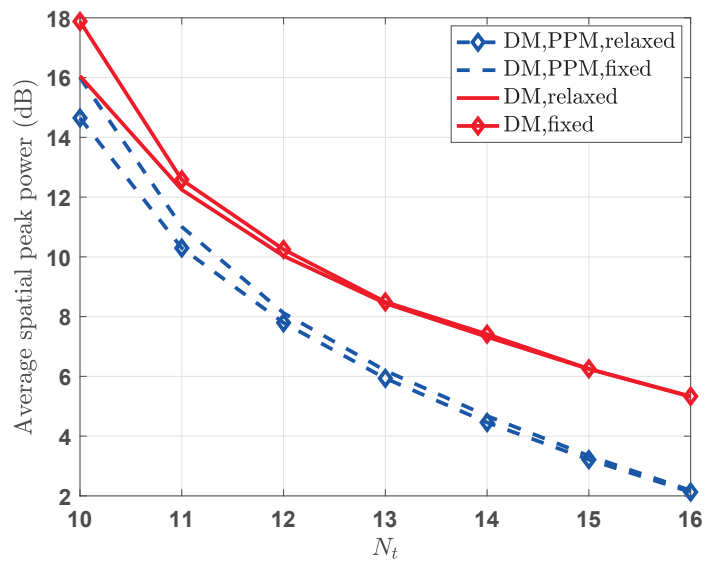

Fig. 3. Average maximum power among the RF chains with respect to $N_{t}$ for the proposed and the benchmark schemes when $S N R=10 \mathrm{~dB}, N_{r}=10$, and $M=16$

where

$$
\mathbf{D}=\left[\begin{array}{c}
\widetilde{\mathbf{H}}_{2}-b_{1} \widetilde{\mathbf{H}}_{1} \\
\widetilde{\mathbf{H}}_{2}-b_{2} \widetilde{\mathbf{H}}_{1}
\end{array}\right], \mathbf{d}=\left[\begin{array}{c}
a_{1} \mathbf{1} \\
-a_{2} \mathbf{1}
\end{array}\right]
$$

The spatial peak power minimization relaxed design in (16) has a linear objective with linear and quadratic constraints which is a convex optimization problem and can be solved using standard methods. To have feasible design problem in both (10) and (16), we assume that $N_{t} \geq N_{r}$.

\section{Simulation Results}

In this part, we present simulations to evaluate the performance of the designed directional modulation transmitter. The examined performance metrics include average transmitter's total power consumption, average spatial peak power, and average symbol error rate (SER) at the receiver. The average of the mentioned metrics is carried out over multiple designed precoders for various data and channel realizations. In all simulations, channels are considered to be quasi static block Rayleigh fading ones generated using i.i.d. complex Gaussian random variables with distribution $\mathcal{C N}(0,1)$ and remain fixed

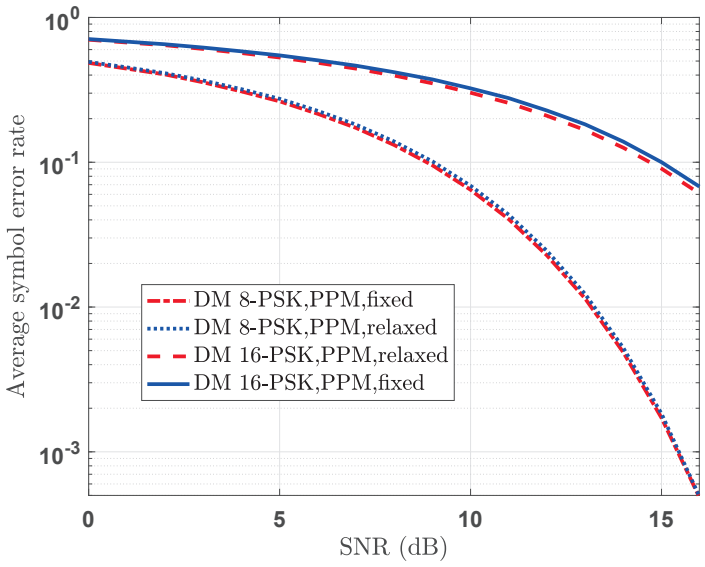

Fig. 4. Average symbol error rate with respect to the required SNR at the receiver for spatial peak power minimization precoder designs with fixed and relaxed phase constraint when $N_{t}=N_{r}=10$.

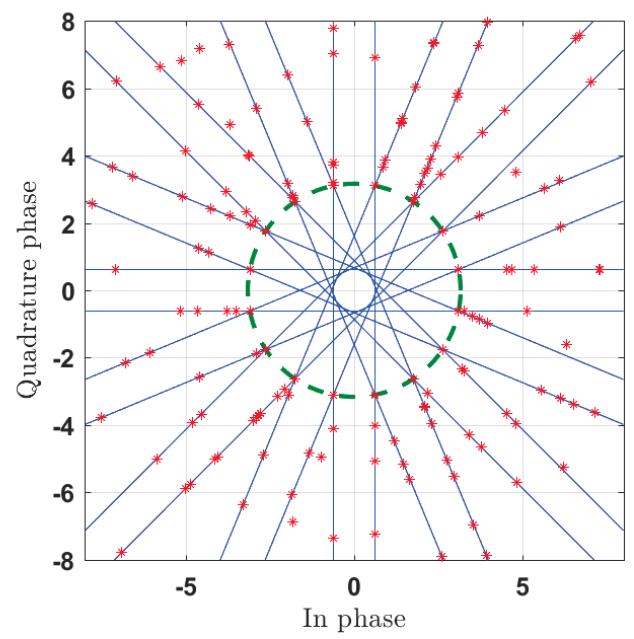

Fig. 5. the distribution of the noise-free communicated symbols in the relaxed spatial peak power minimization design for $N_{t}=N_{r}=500$ and 16-PSK modulation.

during an interval of $N_{r} M$-PSK symbols that are communicated with the receiver. Also, the noise is generated using i.i.d. complex Gaussian random variables with distribution $\mathcal{C N}\left(0, \sigma^{2}\right)$. As the benchmark schemes, we consider the work in [9], which studies fixed and relaxed precoder designs with total transmit power minimization.

In the first scenario, we quantify the power consumption at the transmitter for fixed and relaxed designs with total and spatial peak power minimization criteria in Fig. 2. As we see, the relaxed design reduces the consumed power at the transmitter for a long range of $N_{t}$. This biggest differences between fixed and relaxed designs are $1.28 \mathrm{~dB}$ and $0.84 \mathrm{~dB}$ for total and spatial power minimization problems, respectively, for $N_{t}=N_{r}=10$. As $N_{t}$ grows bigger than $N_{r}$, the system degrees of freedom increases and the power consumption difference between fixed and relaxed designs decreases. Interestingly, we see that the spatial peak power minimization design with relaxed phase consumes less power at $N_{t}=10$ compared to the fixed design with total power minimization 
objective. This illustrates that even with hardware limitation, the spatial peak power minimization design, the relaxed design can results in an more energy efficient transmitter compared to the fixed design.

For the next scenario, we present the average spatial peak power for fixed and relaxed designs with total and spatial peak power minimization objectives. As we see in Fig. 3, the spatial peak power minimization with relaxed design results in a considerably less spatial peak power compared to the fixed design for a long range of $N_{t}$. For example, for $N_{t}=N_{r}=10$, the relaxed design results in $1.4 \mathrm{~dB}$ less average spatial peak power compared to the fixed design. As $N_{t}$ increases, the difference between the average spatial peak power of the fixed and relaxed designs decreases as Furthermore, as $N_{t}$ increases, the difference between the maximum output results in less maximum output power among the RF chains.

The distribution of the communicated symbols at the receiver is shown in Fig. 5. As we see, the optimal precoder design results in many symbols going well above the required SNR, the dashed circle, at the receiver. Hence, by using lower power, it is possible to get lower SER thanks to optimizing the locations of the symbols.

As the last scenario, we derive the SER at the receiver for fixed and relaxed cases of spatial peak power minimization designs. SER with respect to SNR is shown in Fig. 4 for 8 PSK and 16-PSK modulations. We observe that the SER of fixed and relaxed designs are close to each other in relatively low SNR regime and the distance between them increases for relatively high SNR regime. In addition, the SER of the relaxed and fixed designs for 8-PSK modulation are closer compared to those of 16-PSK modulation. The reason is that the symbol detection region shrinks as the modulation order increases and the relaxed design is more likely to create an error. By comparing Figs. 2 and 4 for $S N R=10 \mathrm{~dB}$ and 16-PSK modulation, we see that the power saving for a range of $N_{t}$ is worthy of the loss in SER, especially for $N_{t}=N_{r}$.

\section{CONCLUSIONS}

In this work we designed the optimal symbol-level precoder for a relaxed phase $M$-PSK directional modulation transmitter by minimizing the maximum transmitted power among the RF chains. In contrast to the state of the art, we let the communicated symbols to vary in a predefined relaxed region instead of considering them to be fixed in a point. The simulations demonstrated that relaxing the phase results in less power consumption at the transmitter, especially for close number of transmit and receiver antennas. Interestingly, it was observed that the relaxed spatial peak power minimization design consumes less power than the fixed design with transmit power minimization objective for equal number of transmit and receive antennas. The results showed that relaxed design results in a less maximum transmitted power among the RF chains compared to the fixed design. While the relaxed design reduces the total power consumption and spatial peak power notably, the SER increment due to relaxed design is negligible compared to the power reduction, especially for close values of transmit and receive number of antennas. The power reduction and SER improvement in directional modulation are derived at the expense of designing the precoder for communication of each group of the symbols.

\section{REFERENCES}

[1] Cisco, "Cisco visual networking index: Forecast and methodology, 2015-2020," Cisco, Tech. Rep., 2016. [Online]. Available: http://www.cisco.com/c/en/us/solutions/collateral/serviceprovider/visual-networking-index-vni/complete-white-paper-c11481360.pdf

[2] S. Weinstein and P. Ebert, "Data transmission by frequency-division multiplexing using the discrete fourier transform," IEEE Trans. Commun. Technol., vol. 19, no. 5, pp. 628-634, Oct. 1971.

[3] D. D. Falconer, F. Adachi, and B. Gudmundson, "Time division multiple access methods for wireless personal communications," IEEE Commun. Mag., vol. 33, no. 1, pp. 50-57, Jan. 1995.

[4] Q. Spencer, A. Swindlehurst, and M. Haardt, "Zero-forcing methods for downlink spatial multiplexing in multiuser MIMO channels," IEEE Trans. Signal Process., vol. 52, no. 2, pp. 461-471, Feb. 2004.

[5] N. Sidiropoulos, T. Davidson, and Z.-Q. Luo, "Transmit beamforming for physical-layer multicasting," IEEE Trans. Signal Process., vol. 54, no. 6, pp. 2239-2251, Jun. 2006.

[6] A. Babakhani, D. Rutledge, and A. Hajimiri, "Transmitter architectures based on near-field direct antenna modulation," IEEE J. Solid-State Circuits, vol. 43, no. 12, pp. 2674-2692, Dec. 2008.

[7] M. Daly and J. Bernhard, "Directional modulation technique for phased arrays," IEEE Trans. Antennas Propag., vol. 57, no. 9, pp. 2633-2640, Sep. 2009.

[8] M. Daly, E. Daly, and J. Bernhard, "Demonstration of directional modulation using a phased array," IEEE Trans. Antennas Propag., vol. 58, no. 5, pp. 1545-1550, May 2010.

[9] A. Kalantari, M. Soltanalian, S. Maleki, S. Chatzinotas, and B. Ottersten, "Directional modulation via symbol-level precoding: A way to enhance security," IEEE J. Sel. Topics Signal Process., vol. PP, no. 99, Aug. 2016.

[10] C. Masouros and E. Alsusa, "Dynamic linear precoding for the exploitation of known interference in MIMO broadcast systems," IEEE Trans. Wireless Commun., vol. 8, no. 3, pp. 1396-1404, March 2009.

[11] _ "Soft linear precoding for the downlink of DS/CDMA communication systems," IEEE Trans. Veh. Technol., vol. 59, no. 1, pp. 203-215, Jan. 2010.

[12] M. Alodeh, S. Chatzinotas, and B. Ottersten, "Constructive multiuser interference in symbol level precoding for the MISO downlink channel," IEEE Trans. Signal Process., vol. 63, no. 9, pp. 2239-2252, May 2015.

[13] A. A. M. Saleh, "Frequency-independent and frequency-dependent nonlinear models of TWT amplifiers," IEEE Trans. Wireless Commun., vol. 29, no. 11, pp. 1715-1720, Nov. 1981.

[14] S. K. Mohammed and E. G. Larsson, "Single-user beamforming in large-scale MISO systems with per-antenna constant-envelope constraints: The doughnut channel," IEEE Trans. Wireless Commun., vol. 11, no. 11, pp. 3992-4005, Nov. 2012.

[15] J. Pan and W. K. Ma, "Constant envelope precoding for single-user large-scale MISO channels: Efficient precoding and optimal designs," IEEE J. Sel. Topics Signal Process., vol. 8, no. 5, pp. 982-995, Oct. 2014.

[16] D. Spano, M. Alodeh, S. Chatzinotas, and B. Ottersten, "Per-antenna power minimization in symbol-level precoding," in IEEE Global Commun. Conf. (GLOBECOM), Washington, DC, USA, Dec. 2016.

[17] A. Fehske, G. Fettweis, J. Malmodin, and G. Biczok, "The global footprint of mobile communications: The ecological and economic perspective," IEEE Commun. Mag., vol. 49, no. 8, pp. 55-62, Aug. 2011.

[18] C. Masouros and G. Zheng, "Exploiting known interference as green signal power for downlink beamforming optimization," IEEE Trans. Signal Process., vol. 63, no. 14, pp. 3628-3640, Jul. 2015.

[19] M. Alodeh, S. Chatzinotas, and B. Ottersten, "Energy-efficient symbollevel precoding in multiuser miso based on relaxed detection region," IEEE Trans. Wireless Commun., vol. 15, no. 5, pp. 3755-3767, May 2016.

[20] G. Strang, Introduction to Linear Algebra, 4th ed. WellesleyCambridge Press and SIAM, 2009. 